\title{
Diseño e Implementación de un Sistema de Gestión de la Calidad ISO 9001:2015 en una Universidad Colombiana
}

\author{
Tomás J. Fontalvo y Efraín J. De La Hoz \\ Universidad de Cartagena, Facultad de Ciencias Económicas, Programa Administración Industrial. \\ Campus Piedra de Bolívar, Cartagena. Colombia, (e-mail: tfontalvoh@unicartagena.edu.co; \\ edelahozg@unicartagena.edu.co).
}

Recibido Jul. 21, 2017; Aceptado Sep. 21, 2017; Versión final Nov. 15, 2017, Publicado Feb. 2018

\begin{abstract}
Resumen
En el desarrollo de este trabajo de investigación, se establecen los criterios y estructuras para el diseño e implementación de un sistema de gestión de la calidad, considerando la norma ISO 9001:2015 para una institución universitaria de educación superior en Colombia. Como fundamento teórico para la elaboración de esta investigación se trabajó con los conceptos de sistemas de gestión de la calidad, normalización y los lineamientos de alta calidad para programas académicos. Como resultado de esta investigación se aporta al sector universitario y a la sociedad en general las estructuras operativas, y un programa para el diseño e implementación del sistema de gestión de la calidad en una institución universitaria de educación superior. También se definen las actividades y los responsables asociados al diseño e implementación de la norma ISO 9001: 2015.
\end{abstract}

Palabras claves: sistema de gestión de la calidad; procesos; educación universitaria; gestión del riesgo

\section{Design of a Quality Management System ISO 9001:2015 in a Colombian University}

\begin{abstract}
In the development of this research, the criteria and structures for the design and implementation of a quality management system are established, considering ISO 9001:2015 for a university institution in Colombia. As a theoretical basis for the elaboration of this research the concepts of quality management systems, standardization and high quality guidelines for academic programs were considered. As a result of this research, the university sector and the society in general are provided with operational structures and a program for the design and implementation of the quality management system in a university institution. Also, the activities, and the responsible people associated with the design and implementation of ISO 9001:2015 are defined.
\end{abstract}

Keywords: quality management system; processes; university education; risk management 


\section{INTRODUCCIÓN}

Hasta hace muy poco, la gestión de calidad en las instituciones educativas no era un tema tratado en sus planes de desarrollo. Los gobiernos en su mayoría, daban prioridad a la cobertura, financiación, descentralización, etc como aspectos de mejoramiento del servicio y de desarrollo nacional. Sin embargo, el panorama ha cambiado y la calidad de la educación es un tema de creciente interés en el contexto académico y gubernamental (Guerra y Jaya, 2016). En EE.UU la gestión de calidad tomó importancia en los años 80 debido a la crisis en término de calificaciones de los estudiantes, la financiación y las quejas de empleadores y padres, comenzando implementaciones exitosas en la década de los 90 . Sin embargo en el reino unido el proceso ha sido más lento (Gopal, et al., 2010). Por su parte, Quinn, et al., (2009) a partir de estudios de aplicaciones históricas representativas de técnicas de calidad hallaron resultados positivos sobre a aplicación de las técnicas de calidad en la educación superior.

Las normas ISO surgen en su primera versión en el año 1987 como estándares que definen un sistema de gestión de la calidad. Desde entonces, estas normas se han revisado pasando por versiones en los años 1994, 2000, 2008 y recientemente por la versión 2015. Son las instituciones educativas europeas en la década de 1990, seguidas por las de Estados Unidos y Asia, las que inician su implementación en el contexto de la educación, encontrando grandes dificultades dado su enfoque orientado a la fabricación (Abbadi, et al., 2013). Guerra y Jaya (2016) conceptúan la calidad tomando como referente la norma internacional ISO 9000:2015 como el grado en que un conjunto de características inherentes al servicio educativo cumplen con los requisitos asociados a las necesidades y expectativas de los grupos de interés. De esta manera, la norma ISO 9001: 2015 favorece un enfoque estratégico fortaleciendo el pensamiento basado en el riesgo para planificar y ejecutar acciones dentro de un sistema de gestión calidad que satisfaga de manera equilibrada los requerimientos en la tres dimensiones de la educación superior: docencia, investigación y extensión (Guerra y Jaya, 2016).

Por otra parte, el nuevo modelo de la norma ISO 9001:2015 trae consigo cambios significativos para generar impacto y resultados en los clientes y partes interesadas, cobrando importancia el estudio de contexto, la planificación estratégica, la gestión del cambio y la gestión del riesgo de manera especial, con lo cual se persigue que las organizaciones contextualicen el riesgo en la planificación, implementación, mantenimiento y mejora del sistema. Es importante señalar que algunos autores muestran cómo implementar la norma ISO 9001, y de manera práctica proponen estructuras y actividades para el diseño e implementación de sistemas de gestión de la Calidad en cualquier contexto organizacional (Fontalvo, 2000). Por su parte Calvo, et al., (2006) han analizado como implementar otros estándares de alta excelencia para el mejora-miento de los procesos en la educación superior, lo que facilita la planificación, control y mejoramiento de la gestión, lo que redunda en el cumplimiento de las expectativas de los diferentes actores o grupos de interés.

Calvo, et al., (2005), en su investigación demuestra científicamente como Instituciones de Educación Superior pueden orientar su gestión hacia resultados de excelencia cuando implementan modelos asociados a los sistemas de gestión de la calidad. Así mismo Calvo y Criado, (2005), también señalan como la planificación estratégica y la política de calidad debe alinearse con los procesos y procedimientos, especialmente los procesos docentes, para impactar de manera positiva en los estudiantes y a los grupos de interés que los reciben al momento de egresar de los programas. Así mismo, Naghi, et al., (2015), muestran al igual que esta investigación que cuando se implementa un modelo de gestión de la calidad como el EFQM junto con estándares que evalúan el desempeño académico como el modelo Joghatay, se evidencian mejoras significativas en las instituciones en donde se aplique estos tipos de estándares. Por su parte, Acosta, et al., (2017) muestran como la implementación de sistemas de información estratégico contribuyen al perfeccionamiento de la gestión universitaria, como también se analiza en esta investigación. En este sentido Garbanzo (2016) señala que "la gestión de la educación posee un rol fundamental en la conducción eficiente de las organizaciones educativas, desarrollándolas como organizaciones inteligentes, de manera que alcancen altos estándares de calidad en el servicio que ofrecen, traducido en una mejor formación según la población meta a la cual se dirige".

Leal, et al., (2016), señalan que cuando se revisan la aplicación del modelo ISO 9001:2015, frente a modelos de calidad para Instituciones de Educación Superior en América Latina, se puede observar que el modelo de la norma muestra correspondencia con las exigencias legales y contribuye con la mejora continua del proceso de formación de los estudiantes. En este mismo orden de ideas Alonso, et al., (2013) en su investigación muestran la importancia de articular las áreas de resultados claves, el direccionamiento estratégico y los procesos sustantivos así como los planes de resultados de profesores y trabajadores de la institución universitaria.

Hoy en día las nuevas condiciones que ha generado la globalización y en especial la comunicación a través de las nuevas tecnologías de la información requieren de sistemas de gestión de la calidad estandarizados, 
efectivos y capaces de responder a las necesidades cambiantes que los clientes y las partes interesadas exigen. En este sentido, la implementación de sistemas de gestión de calidad ajustado a las normas ISO 9001 en procesos claves, estratégicos y de apoyo en las Instituciones de Educación Superior es fundamental para su direccionamiento estratégico (Tlapa, et al,. 2009). Según Yzaguirre (2005), los sistemas de gestión de calidad en las organizaciones educativas traen consigo una mejor gestión de sus procesos sustantivos de docencia, investigación y extensión, mejor control y medición de los procesos, mayor interés y compromiso de los trabajadores lo cual supone mayor motivación y productividad. De manera que se mejore su nivel de satisfacción y por ende se logre mayor fidelización (De la Hoz, et al., 2017). Por lo anterior, las estructuras propuestas de un sistema de gestión de la calidad para las universidades, en esta investigación permiten que las Instituciones de Educación Superior respondan desde un estudio de contexto riguroso que identifique los riesgos y como resultado de este análisis gestionen los potenciales riesgos que restrinja el cumplimiento del direccionamiento estratégico de la institución de educación superior.

Adicionalmente las nuevas condiciones del mercado exigen a las instituciones universitarias, que se gestione el cambio y el conocimiento para suplir las necesidades y expectativas de las partes interesadas. En este sentido Garbanzo (2012) señala que la sociedad actual espera de las instituciones de educación una capacidad de respuesta en forma competitiva a la sociedad del conocimiento, asegurando así su desarrollo y sostenibilidad social, lo cual requiere un proyecto educativo y social más inclusivo. De lo anterior se evidencia la necesidad de normalizar todas las actividades de la organización, alineadas con el direccionamiento estratégico, con el propósito de acceder a niveles superiores de calidad que redunden en la construcción y dinámica de una cultura de calidad. Por tal razón se requiere diseñar e implementar sistemas de gestión de la calidad que garantice lo anterior y de esta forma poder competir en el contexto de la entidad universitaria. Por lo anterior se hace necesario entonces, el desarrollo de procesos de planificación, control y mejoramiento de sistemas de gestión de la calidad en las universidades que especifiquen las etapas, recursos, intencionalidades y metas para la implementación efectiva de éstos en toda la institución, de manera que se faciliten a la comunidad académica los métodos para desarrollar las actividades de forma consistente a través del tiempo para la satisfacción de necesidades de las partes interesadas.

A partir de los planteamientos anteriores, surgen las siguientes preguntas ¿Cuáles son los requisitos y criterios asociados a los estándares de Calidad relacionados con la Norma ISO 9001:2015?, ¿Cómo establecer una estructura operativa y un programa para el diseño e implementación de un sistema de gestión de la calidad considerando los lineamientos de la norma ISO 9001: 2015?, ¿Cuáles son las necesidades específicas propias de las Instituciones de Educación Superior para implementar un sistema de gestión de la calidad?, ¿ Cuáles son las herramientas de calidad operativas específicas requeridas en una universidad para diseñar e implementar un sistema de gestión de la calidad en una institución universitaria?

De lo anterior, surgen como intencionalidades investigativas de este trabajo, el mejoramiento de los procesos y la satisfacción de los grupos de interés en el contexto de las entidades educativas que implementen un sistema de gestión de la calidad con base en la norma ISO 9001:2015, por lo que en esta investigación se propone: i) Un marco lógico de la norma ISO 9001:2015; ii) Un programa para el diseño e implementación de un sistema de gestión de la calidad, en donde se asignen los responsables, tiempos, recursos, propósitos y metas para el diseño del sistema de gestión de la calidad en las instituciones universitarias; iii) Diseñar las estructuras operativas del sistema de gestión de la calidad que garanticen la estandarización de los procesos en las Instituciones de Educación Superior; y iv) Establecer las actividades y responsables que garanticen la implementación del sistema de gestión de la calidad en las Universidades.

\section{METODOLOGÍA}

Para el desarrollo de este trabajo de investigación se contextualizó como objeto de estudio las Instituciones de Educación Superior y se tomaron como referentes los lineamientos de calidad establecidos por el Ministerio de Educación Nacional (MEN) de Colombia y la Norma ISO 9001:2015, lo que conllevó a establecer componentes y requisitos a partir del cual se estructuró la matriz de marco lógico del Sistema de Gestión de la Calidad (ver Fig. 1). La propuesta en términos de estructura operativa y del programa para el diseño e implementación del sistema de gestión de la calidad partió de un análisis racional del estándar de Calidad ISO 9001:2015, definir las variables y componentes asociados a las estructuras del sistema de gestión de la calidad y el plan para la implementación de este, relacionados con las actividades y responsables que permitan a las instituciones universitarias realizar un estudio de contexto que posibilite evaluar los riesgos y establecer el direccionamiento estratégico, para finalmente direccionar los procesos y procedimientos necesarios para generar satisfacción e impacto en los contextos donde se implemente esta propuesta. Lo anterior gestionando el conocimiento y el cambio en las organizaciones educativas analizadas en este estudio.

La esencia de la ciencia en esta investigación, estuvo soportada en un análisis racional por parte de los investigadores, que posibilitó la construcción de objeto de estudio para el mejoramiento de las Instituciones 
de Educación Superior. Así mismo la lógica del método partió de un análisis del estándar de calidad, por lo que se hizo necesario analizar las dimensiones, requisitos y herramientas de estructura operativa y criterios para el diseño e implementación del sistema de gestión de la calidad a partir de la cual se elaboró la propuesta de variables del sistema de gestión de la calidad, así como el establecimiento del plan para el diseño e implementación de este en las Instituciones de Educación Superior.

El análisis racional, se fundamentó, validó en las experiencias y prácticas de los investigadores, en el acompañamiento y direccionamiento de procesos de diseño e implementación de sistemas de gestión de la calidad en instituciones y programas universitarios en Colombia, trabajos y estudios realizado por los autores en diferentes Instituciones de Educación Superior, así como el resultado de las experiencias de los investigadores como pares evaluadores del Ministerio de Educación Nacional MEN realizadas en diferentes universidades de Colombia y en el acompañamiento y participación de programas reconocidos en calidad de 10 Instituciones de Educación Superior en Colombia.

\section{DISCUSIÓN Y RESULTADOS}

Con el fin de darle respuestas a los interrogantes de esta investigación se estudió y analizó la norma ISO 9001: 2015 observándose requisitos y dimensiones exigidos en los procesos de certificación de calidad de una organización bajo la norma ISO 9001: 2015. Como resultado, se diseñaron las estructuras que forman el sistema de gestión de la calidad, junto con el programa de implementación, para lo cual se definieron las actividades y responsables asociadas al desarrollo de un sistema de gestión de la calidad en Instituciones de Educación Superior. Para lo anterior se contextualizó la norma ISO 9001: 2015 a los requerimientos asociados a los lineamientos de acreditación de alta calidad de las Instituciones de Educación Superior establecidos por el MEN de Colombia. Así mismo se establecieron directrices para desarrollar las estructuras operativas, de planificación y control en las Instituciones de Educación Superior con el objetivo de alcanzar altos estándares de calidad, con lo cual se proporcionan criterios de dirección para la toma de decisiones, por parte de los directivos de este tipo de instituciones Universitarias.

En la Fig. 1 se plantean los elementos y lineamientos mediante una matriz de marco lógico que estructura el sistema de gestión de la calidad y a su vez direcciona la elaboración del programa para el diseño e implementación del sistema integrado de gestión considerando los referentes asociados a la Norma ISO 9001:2015.
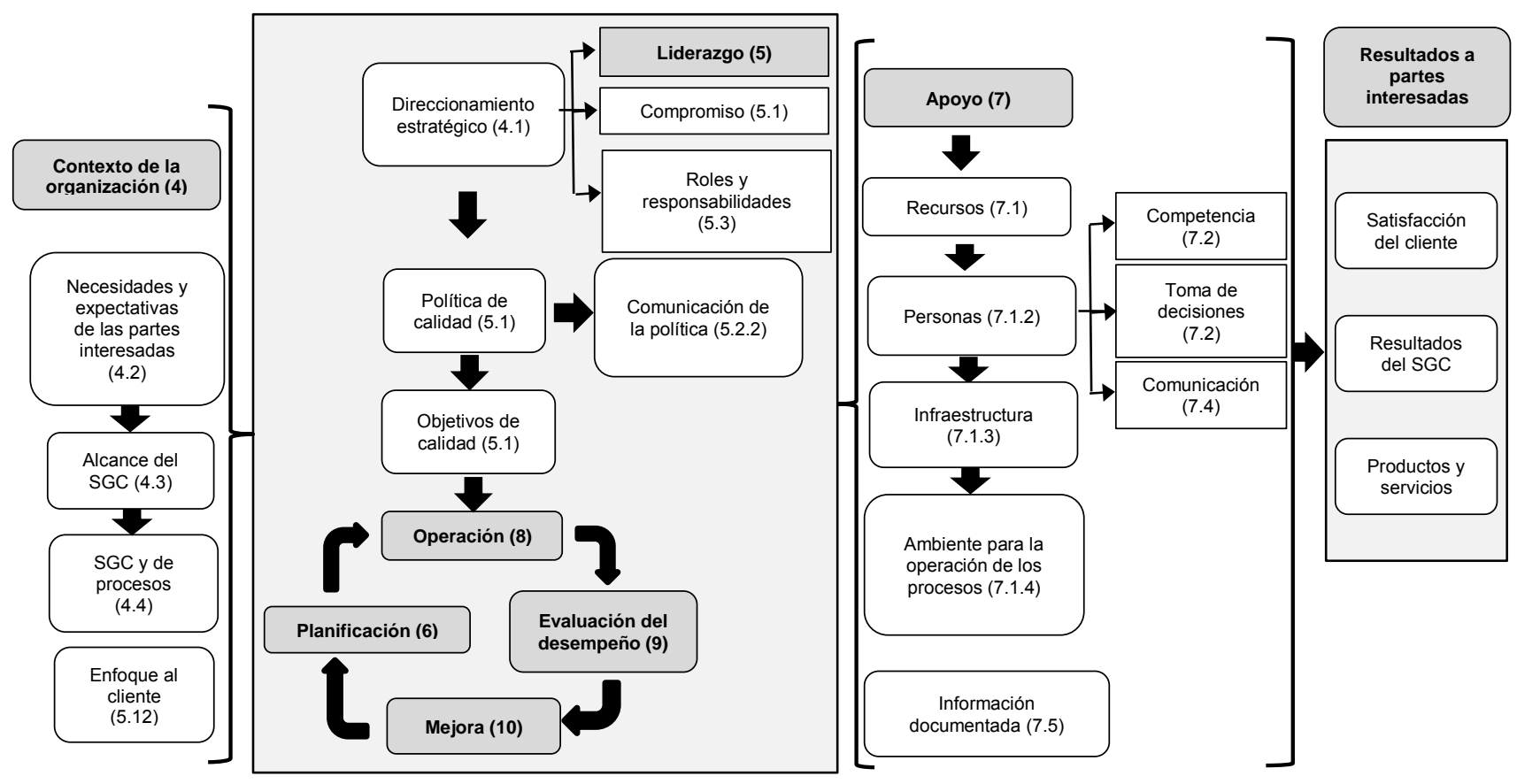

Fig. 1: Marco lógico de un Sistema de Gestión de la Calidad Con base en la norma ISO 9001: 2015

De esta manera, con la estructura asociada al sistema de gestión de la calidad con base en la norma ISO 9001:2015 y su contextualización en Instituciones de Educación Superior o universitarias, se aporta al sector de la educación superior un programa para el diseño e implementación de un sistema de gestión de la calidad, especificando elementos, componentes y estructuras operativas que garantizan la gestión del cambio y el conocimiento de estas para satisfacer las necesidades de los clientes así como de las partes interesadas para generar un impacto en el contexto donde se implemente el modelo propuesto. En este sentido, García y Calvo, 
(2009) señalan la importancia de ciertos factores asociados al sistema de gestión de la calidad que hacen de estos, procesos exitosos cuando se implantan. Para la operacionalización de la norma ISO 9001:2015 considerada a partir de la matriz de marco lógico propuesta en la Fig. 1, se establecieron macro actividades para implementar la norma en una institución universitaria, las cuales se sintetizan en las actividades que se discuten en lo que sigue.

Diseño e implementación del Sistema de Gestión de la Calidad: A continuación se presentan un conjunto de actividades asociadas con el diseño e implementación de un sistema de gestión de la Calidad para un programa académico de educación superior.

Estudio de contexto de la organización (4): Inicialmente se debe definir un estudio de contexto que permita establecer necesidades y expectativas de las partes interesadas de la institución, para lo cual es necesario establecer necesidades de la región y el país donde la institución interviene, perfiles de los estudiantes que entran, perfiles de los egresados y precisar las competencias a desarrollar en el contexto social donde intervendrán. Lo anterior debe estar asociado con la evaluación de la gestión del riesgo relacionado con las necesidades y expectativas a cumplir en las partes interesadas de la organización donde se implementa el sistema de gestión de la calidad propuesto. Una manera concreta de abordar puede ser la realización de un análisis DOFA (Debilidades, Oportunidades, Fortalezas y Amenazas) por la facilidad y practicidad para realizar este, como lo plantea Fontalvo, (2000).

Necesidades y expectativas de las partes interesadas. En este sentido es importante identificar los grupos de interés o partes interesadas de tal forma que se puedan identificar las necesidades de formación y competencias requeridas por los estudiantes de los diferentes programas de la universidad para garantizar la pertinencia y oportunidad en el desarrollo de las competencias propias de su formación. Lo que se constituyen en un referente de revisión, seguimiento y actualización de la información de tal manera que la universidad se posesione y consolide en contexto donde presta sus servicios educativos.

Direccionamiento estratégico y legal de la institucional: Para esto es necesario reflexionar e interpretar el direccionamiento estratégico institucional de la universidad donde va a implementar. Para lo cual se requieren como actividades mínimas: El diseño del plan estratégico universitario alineado con el estudio de contexto de la Universidad, con sus objetivos, elaboración del Proyecto Educativo Institucional PEI, el estatuto organizacional, el estatuto de investigación, el estatuto profesoral, el reglamento de estudiantes, el reglamento de Bienestar Universitario, la estructura organizacional, el plan de desarrollo de la universidad, el sistema institucional de investigaciones, reuniones con grupo de directivos institucionales, lo cual requiere un análisis de documentos, talleres y conversatorios con los responsables, como son equipo operativo del proyecto, una comisión del proyecto y grupo directivo de la institución.

Estado actual del sistema de gestión de calidad a nivel institucional: Esta actividad es fundamental para la organización, por lo que se requiere realizar un diagnóstico del sistema de gestión de la universidad analizada, con lo cual establecer el diseño conceptual del mismo de acuerdo a las carencias y características de la institución, frente a las exigencias del direccionamiento estratégico institucional y requerimientos de la norma ISO 9001:2015. Lo anterior permite proponer recomendaciones preliminares relacionadas con ajustes e implementaciones institucionales, por lo que se hace necesaria la revisión de documentos, procesos y procedimientos institucionales, así como sesiones de discusión sobre dichos documentos, para finalmente consolidar un informe del estado actual de los procesos, procedimientos y recomendaciones preliminares.

Como metodología se requiere una revisión y análisis documental de los lineamientos establecidas en las Condiciones de alta calidad del MEN-Colombia (o autoridad responsable en cada país), para la apertura y funcionamiento de instituciones y programas, los procesos existentes previos, el sistema información, informes de avance proceso de autoevaluación con fines de mejora continua de la calidad desarrollados por la institución, criterios de evaluación, con lo que se puede evaluar el nivel de cumplimento de la organización frente la norma ISO 9001. Con lo anterior, se establecen las estructuras existentes del sistema y un documento diagnóstico y recomendaciones asociado al sistema intervenido

Liderazgo (5): La alta dirección debe demostrar liderazgo y compromiso con respecto a los propósitos del sistema de gestión, asumiendo responsabilidades y obligaciones frente a los compromisos que este genere, garantizando que se establezca la política de calidad y los objetivos de calidad, garantizando que se integren los requisitos de la norma a los intereses y naturaleza propia de la universidad donde se implementa el sistema de gestión, previendo un enfoque de la gestión del riesgo y de procesos, gestionando los recursos necesarios para la consolidación de sistema, comunicado la eficacia del sistema, asegurando que la eficacia del sistema de gestión y mejoras. Comprometiendo, dirigiendo y apoyando las personas de tal forma que estas asuman sus roles que garanticen la eficacia del sistema de gestión del a calidad. 
Planificación (6): Uno de los nuevos planteamientos del modelo de la Norma ISO 9001:2015 está asociado con la Gestión del riesgo y las oportunidades de mejora. Para lo anterior es importante que las entidades educativas identifiquen las necesidades y oportunidades de las partes interesadas. Así como los riesgos de estas y seleccionen un modelo para valorar los riesgos de tal forma que se puedan mitigar los riesgos en la institución universitaria.

En concordancia con lo anterior, como resultado del estudio de contexto de la organización se genera un buen direccionamiento estratégico. Esto permite identificar las necesidades y expectativas de las partes interesadas que a su vez contribuye con el poder identificar y gestionar el riesgo para cumplir con las necesidades y expectativas de los estudiantes, el sector empresarial, el estado y la comunidad universitaria lo que a la postre posibilita satisfacer las necesidades de las partes interesadas y genera certidumbre asociada a los propósitos institucionales. Mitigación de los riesgos que se traduce en la mejora de la prestación del servicio educativo mediante la valoración del riesgo en la satisfacción de necesidades y expectativas. Ver Tabla 1.

Tabla 1: Valoración del riesgo de las necesidades y expectativas de las parte interesadas

\begin{tabular}{|l|c|c|c|c|c|c|c|}
\hline Proceso & $\begin{array}{c}\text { Parte } \\
\text { interesada }\end{array}$ & $\begin{array}{c}\text { Necesidades y } \\
\text { expectativas }\end{array}$ & Riesgo & $\begin{array}{c}\text { Probabilidad } \\
(P)\end{array}$ & $\begin{array}{c}\text { Exposición } \\
(E)\end{array}$ & $\begin{array}{c}\text { Consecuencia } \\
(C)\end{array}$ & $\begin{array}{c}\text { Dimensión } \\
P^{\star} E^{\star} C\end{array}$ \\
\hline & & & & & & & \\
\hline
\end{tabular}

Lo anterior permite gestionar el riesgo no solo como se mencionó en el ítem del estudio de contexto de la organización, sino también a nivel de la estructura operativa del sistema de gestión de la calidad, específicamente en los procesos. Por otro lado, la planificación requiere también que la institución universitaria establezca sus objetivos de calidad, los cuales deben integrare a la política de calidad y deben ser medibles, tener en cuenta los requisitos asociados a la norma, pertinentes al servicio educativo, comunicarse y actualizarse periódicamente. Así mismo, la planificación de los cambios requiere que la universidad una vez implementado el sistema de gestión de la Calidad, considere y responda los siguientes interrogantes. ¿Cuál es la intencionalidad de los cambios y su efecto?, ¿Cómo afecta la integralidad del sistema de Gestión?, ¿Existe la disponibilidad de recursos para realizar los cambios?, ¿Frente a los cambios como se realizará la asignación o reasignación de responsabilidades y autoridades?

Apoyo (7): Para el diseño y consolidación de un sistema de gestión de la Calidad en una institución universitaria es fundamental garantizar las capacidades y condiciones de los recursos internos existentes pertinentes a los servicios educativos que se prestan. Así como también el talento humano para la operación y control de los procesos sustantivos como son docencia, investigación, extensión e internacionalización, formados a nivel de Maestría y doctorados. De igual forma se requiere una infraestructura adecuada y pertinente a las necesidades de formación que garanticen las necesidades y expectativas de los grupos de interés. Otra variable importante del nuevo modelo de la norma tiene que con la gestión del conocimiento al interior de la institución educativa, para lo cual es importante garantizar los conocimientos que aseguren el desarrollo de los procesos para la prestación de los servicios de los programas de Pregrado, Maestrías y doctorados, para el caso del as instituciones que desarrollen este tipo de formación.

Frente a las condiciones y necesidades cambiantes delas partes interesadas es importante que la entidad educativa gestione el conocimiento tácito y explicito (Documentación del sistema), para lo cual se hace necesario que se desarrollen actividades asociadas a la socialización del conocimiento, La interiorización del conocimiento, la exteriorización del conocimiento y la combinación del conocimiento existente de tal forma que se preserve, mantenga y cree nuevo conocimiento que le garantice a la institución educativa ser competitiva y pueda ofertar programas pertinentes que respondan a las necesidades de los usuarios.

Operación del sistema de gestión de la calidad (8): Identificación de los macro procesos institucionales: Una vez revisado y analizado se realizará un inventario de los macro procesos institucionales que se deben llevar a cabo con base en el direccionamiento estratégico y los procesos requeridos por la organización y la norma ISO 9001:2015, así como clarificar las responsabilidades de los actores institucionales. Para esto se realizarán, reuniones con directivos institucionales, a través de sesiones de trabajo en grupo y talleres con el equipo operativo del proyecto y comisión del proyecto.

Diseño del modelo del sistema de gestión de calidad: Esto implica la estructuración del modelo del sistema de gestión de calidad, acorde con las necesidades y características propias de cada institución universitaria, por lo que se requiere que este contemple fortalezas propias de la organización y las exigencias del modelo de la norma ISO 9001:2015 alineado con el direccionamiento estratégico institucional. Para lo cual es necesario reuniones con grupos que permitirá consolidar el diseño del modelo de sistema de calidad específico para la institución donde se implemente este modelo que responda a las partes interesadas de la universidad donde se implemente la norma ISO 9001:2015 
Estructuración y documentación del manual de procesos y procedimientos del sistema de gestión de la calidad: Una vez definido el modelo del sistema y su estructura general se procederá a elaborar los manuales de procesos y procedimientos del sistema de gestión de la calidad de la calidad. Alonso (2014), señala la importancia de éstos, para la creación de valor y planeación del sistema una buena documentación que soporte la gestión por procesos. Lo cual requiere el desarrollo de las siguientes actividades al interior de la institución universitaria: selección del equipo de trabajo de campo, diseño de instrumentos y documentos, formación del equipo de trabajo, levantamiento de la información de campo, análisis y diseño de los procesos existentes, faltantes y mejorados, elaboración de los manuales de procesos y procedimientos propios de la entidad educativa, manual de gestión de riesgos, matriz de identificación de riesgos, procedimiento de gestión del cambio, procedimiento de gestión del conocimiento, procedimiento de gestión de la mejora y manual de calidad, como lo precisan los investigadores Fontalvo, et al., (2011)

Para lo anterior se requieren las siguientes actividades: entrevistas individuales y grupales, capacitación y entrenamiento, diseño de instrumentos, reuniones de trabajo y validación de información, organización y sistematización de información, análisis de documentos y redacción de documentos. Como responsables se necesita: un equipo operativo del proyecto y un equipo de trabajo de campo. Con esto se posibilita la consecución de la meta establecida, lo que implica la elaboración y entrega del $100 \%$ de los manuales de procesos, el manual de calidad y procedimientos. En este sentido Rodríguez, et al., (2012), demuestran a través de un modelo explicativo que existe una tendencia que vincula significativamente la calidad de la información con la calidad de las universidades. Por lo que todas estas herramientas asociadas a la documentación del sistema de gestión de la calidad, contribuyen significativamente con la calidad en las instituciones educativas.

Evaluación del desempeño del sistema de Gestión de la Calidad (9): Auditoría para la consolidación del sistema de gestión de la Calidad en las instituciones Universitarias: Una vez diseñado el sistema de gestión, el paso siguiente está asociado con el desarrollo de las auditoría de primera parte con el fin de evaluar y consolidar el sistema de gestión para la realización de las auditoría de segunda parte o pasar directamente a las auditoría de tercera parte o también conocidas como auditoría de certificación del sistema de gestión de la calidad. La auditoría se desarrollará considerando las etapas de planeación, preparación, ejecución y seguimiento y cierre de la auditoría. Que garanticen la eficacia del sistema para cumplir las necesidades del as partes interesadas. Adicionalmente a los procesos de auditoría que nos permite evaluar el sistema de gestión de la calidad en la institución educativa también es necesario analizar como lo plantea Gómez y Sánchez (2013), medir la calidad con indicadores. De tal forma que se tenga una percepción más amplia del contexto objeto de evaluación.

Mejora del sistema de gestión del a Calidad (10): Desde esta perspectiva es necesario que la universidad identifique las oportunidades de mejora en términos de llenar las necesidades y expectativas de las partes interesadas que se traduzca en resultados organizaciones reduciendo el riesgo en los procesos de la institución. Así como oportunidades de mejora que se derivan de las no conformidades detectadas y de las acciones correctivas resultantes de las Auditorías de calidad.

Organización administrativa del proyecto y responsabilidades: Para llevar a cabo el proyecto de diseñar un sistema de gestión de la calidad en una institución universitaria, se requiere una estructura y definición de roles de personal para el desarrollo del proyecto, describiendo a continuación las responsabilidades básicas que deben asumir las personas que se designen en los diferentes cargos o roles del proyecto.

Rresponsabilidades básicas de los cargos de la organización Técnico- Operativa del proyecto: Comisión del proyecto: Ejercer la veeduría de la ejecución del proyecto y aprobar en primera instancia los avances, informes y documentos finales resultantes del proyecto. Ser interlocutor ante la rectoría y órganos de dirección institucional.

Director técnico: Es el responsable de la planeación, dirección, coordinación, seguimiento, evaluación y control de los aspectos técnicos del proyecto, relacionados con el diseño del Sistema de Gestión de Calidad y la elaboración y documentación de los procesos, procedimientos e instructivos.

Grupo asesor y de apoyo técnico: Como responsabilidad le corresponde la asesoría y apoyo técnico al Director Técnico del proyecto. Se propone que esté conformado por un Asesor especialista en los temas técnicos a desarrollar y dos (2) profesionales con experiencia en éste tipo de proyectos. Este grupo participará activamente en el diagnóstico inicial, la definición y valoración de riesgos, en el diseño del modelo del sistema de Gestión de la Calidad, el análisis crítico y mejora de los procesos y procedimientos.

Coordinación administrativa y logística: Este cargo responde por la coordinación, seguimiento y apoyo administrativo y logístico para asegurar y garantizar el normal desarrollo y ejecución del plan de trabajo y cronograma del proyecto. 
Coordinadores de grupos de trabajo de campo: Adicionalmente se requiere un cargo que responda por coordinación, supervisión, seguimiento y análisis y revisión técnica preliminar del trabajo de campo del grupo de profesionales expertos y conocedores del tema de sistemas de gestión de la calidad, que participarán en el diseño e implementación del sistema de gestión de la calidad

Equipo de trabajo de campo: Este equipo de profesionales será el responsable de la documentación y análisis preliminar en el diseño e implementación del sistema de gestión de la calidad, los procesos, procedimientos e instructivos que se deben elaborar y responden por la documentación del proyecto, de acuerdo con el modelo del sistema diseñado y con base en la capacitación impartida por el equipo técnico del proyecto. Este equipo de trabajo estará conformado por profesionales expertos y conocedores del tema en sistemas de gestión de la calidad.

\section{CONCLUSIONES}

La presente investigación constituye una propuesta para desarrollar sistemas de gestión de calidad en la educación que contribuyan al logro de los objetivos misionales de docencia, investigación y extensión. Sin embargo, otros autores a nivel internacional también han desarrollado investigaciones en el área de la calidad en la educación desarrollando propuestas, metodologías o modelos para la implementación de sistemas de gestión de la calidad abordados desde diferentes perspectivas. Saktivhivel,et al., (2005) establecen un modelo de excelencia de calidad académica relacionando su implementación con la satisfacción en el rendimiento académico del estudiante. Lundquist (2006) presenta directrices sobre la implementación de sistemas de calidad en la educación atendiendo la norma ISO 9000 pero sin proponer una metodología estructurada para tal fin. Quinn, et al., (2009) identifican técnicas para evaluar la mejora de la calidad en la educación superior. Gopal, et al., (2010) proponen principios, conceptos básicos y criterios del éxito para la excelencia para evaluar la calidad en los procesos de la educación superior en el Reino Unido.

Del análisis y discusión de los resultados, también se puede concluir: 1) En este trabajo se muestra cómo a partir de estándares de calidad con reconocimiento mundial como son los de la norma ISO $9001: 2015$ y referentes de calidad específicos (Como los lineamientos de acreditación de alta calidad del MEN-Colombia), se puede responder a las necesidades y expectativas de los clientes en una institución universitaria. Siendo pertinente con las necesidades de formación de los estudiantes para que pueden intervenir en la sociedad de forma exitosa y responder a las necesidades de las partes interesadas. 2) Es determinante que se gestione el conocimiento asociado a todos los procesos, procedimientos, instructivos y se desarrollen panoramas de riesgos de tal forma que se tomen las acciones que contribuyan a la consecución de los objetivos y metas ligadas al direccionamiento estratégico del sistema de gestión de la calidad.

\section{REFRENCIAS}

Acosta, L., Becerra, F., y Jaramillo, D. Sistema de Información Estratégica para la Gestión Universitaria en la Universidad de Otavalo (Ecuador). Formación Universitaria, 10(2), 103-112. (2017)

Agus, A. The Structural Linkages Between TQM, Product Quality Performance and Business Performance: Preliminary Empirical Study in Electronics Companies. Singapore Management Review, 27(1), 87-105 (2005)

Alonso, A., Michelena, E. y Alfonso, D. Dirección por procesos en la Universidad. Rev. Ingeniería Industrial, 34(1), 87-95 (2013)

Alonso, C. Orientaciones para implementar una gestión basada en procesos. Rev. Ingeniería Industrial, 35(2), $159-171(2014)$

Calvo, A., Leal, A. y Roldán, J. Using enablers of the EFQM model to management institutions of higher education, Quality Assurance in Education, 14 (2), 99-122 (2006)

Calvo, A., Leal, A. y Roldán, J. Relationships between the EFQM model criteria: a study in Spanish universities. Total Quality Management, 16(6), 741 -770 (2005)

Calvo, A., Criado, F. Análisis de la validez del modelo europeo de excelencia para la gestión de la calidad en instituciones universitarias: un enfoque directivo. Revista Europea de Dirección y Economía de la Empresa, 14(3), 41-58 (2005)

De la Hoz, E., López, L. y Pérez, L. Modelo de gestión de relaciones con los clientes en empresas de Consultoría. Revista Investigación e Innovación en Ingenierías, 5(2), 46-77 (2017) 
El Abbadi, L., Bouayad, A. y Lamrini, M. ISO 9001 and the Field of Higher Education: Proposal for an Update of the IWA 2 Guidelines. Quality Approaches in Higher Education, 4(2), 14-19 (2013)

Fontalvo, H. Herramientas efectivas para el diseño e implantación de un sistema de gestión de la calidad ISO 9000: 2000, Corporación para la gestión del conocimiento ASD 2000 (2000)

Fontalvo, H., La gestión avanzada de la calidad: metodologías eficaces para el diseño, implementación y mejoramiento de un sistema de gestión de la calidad, Corporación para la gestión del conocimiento ASD $2000(2000)$

Fontalvo, H. y Morelos, J. Análisis de los factores determinantes de la cultura organizacional en el ambiente empresarial, Entramado, 10 (1), 96-105 (2014)

Fontalvo, H, Quejada, R. y Puello J. La Gestión del conocimiento y los procesos de mejoramientos, Dimensión Empresarial, 9 (1), 80-87 (2011)

García, F. y Calvo, A. Factores clave y rendimiento en empresas españolas con sistemas de gestión de la calidad. Revista Europea de Dirección y Economía de la Empresa, 18(2), 107-126 (2009)

Garbanzo, G. Desarrollo organizacional y los procesos de cambio en las instituciones educativas, un reto de la gestión de la educación. Revista Educación, 40(1), 67-87 (2016)

Garbanzo, G. Educación superior pública en América Latina: características y desafíos. Revista GUAL, 5(1), 216-227 (2012)

Gómez, H. y Sánchez, V. Indicadores cualitativos para la medición de la calidad en la educación. Educación y Educadores, 16(1), 9-24 (2013)

Gopal, K., Abdul, M. y Bin, T. Total quality management in UK higher education institutions. Journal Total Quality Management, 10(1), 129-153 (2010)

Guerra, R. Jaya, A. "El papel de los Stakeholders en la gestión de la calidad universitaria: el enfoque de la ISO 9001:2015", Revista Caribeña de Ciencias Sociales, 5(12), 1-15 (2016)

Leal, L., Reyes, R., Domínguez, J. y González, A. Correspondencia del Modelo de gestión de la calidad de la Educación Superior en Cuba con la serie ISO 9000. Revista Dilemas Contemporáneos: Educación, Política y Valores, 3(11), 1-21 (2016)

Lundquist, R. Quality systems and ISO 9000 in Higher education. Journal Assessment \& Evaluation in Higher education, 22(2), 159-172 (2006)

Naghi, A., Mohammad, M. Afrassiabi, R. A study of the relationship between the EFQM organizational excellence model and the performance of Joghatay Office of Education. Journal of Applied Environmental and Biological Sciences, 5(10S), 631-639 (2015)

Quinn, A., Lemay, G., Larsen, P. y Johnson, D. Service quality in higher education. Journal Total Quality Management \& Business Excellence, 20(2), 139-152 (2009)

Rodríguez, E., Fleet, N., Pedraja, L. y Rodríguez, J. Efecto de la calidad de la información sobre la acreditación institucional: un estudio exploratorio en universidades chilenas. Ingeniare. Rev. Chil. Ing., 20(3), 368-375 (2012)

Saktivhivel, P., Rajendran, G. y Raju, R. TQM implementation and students' satisfaction of academic performance. The TQM Journal, 17(6), 573-589 (2005)

Tlapa, D., Limón, J. y Báez, Y. Gestión de la calidad y el medio ambiente en Instituciones de Educación Superior mediante integración de ISO 9001 e ISO 14001. Formación Universitaria, 2(2), 35-46. (2009)

Yzaguirre, L. Calidad educativa e ISO 9001:2000 en México. REICE - Revista Electrónica Iberoamericana sobre Calidad, Eficacia y Cambio en Educación, 3(1), 421-431 (2005) 
